\title{
Epidemiological study of HLA and GM in rheumatoid arthritis and related symptoms in an open Dutch population
}

\author{
B. M. DE JONGH, ${ }^{1}$ L. K. J. VAN ROMUNDE, ${ }^{2}$ H. A. VALKENBURG ${ }^{2}$ \\ G. G. DE LANGE, ${ }^{3}$ AND J. J. VAN ROOD 1
}

From the 'Department of Immunohaematology and Blood Bank, University Hospital Leiden, Leiden; the ${ }^{2}$ Department of Epidemiology, Erasmus University of Rotterdam, Rotterdam; and the ${ }^{3}$ Central Laboratory of The Netherlands Red Cross Blood Transfusion Service and Laboratory for Experimental and Clinical Immunology, University of Amsterdam, Amsterdam, The Netherlands

SUMMARY This report deals with the question of whether or not the established association of HLA-DR4 with rheumatoid arthritis (RA) can also be detected in cases of RA as diagnosed in a population survey. For this purpose 6584 persons older than 19 years living in a single community in The Netherlands were investigated for the presence of rheumatoid arthritis and related abnormalities. After five years 83 patients with RA, 30 with only erosive abnormalities on radiological examination (Rad), and 48 with only rheumatoid factor (RF) were reinvestigated and typed for HLA and allotypes of immunoglobulin $\mathrm{G}$ heavy chain (GM). On the classification of the initial survey no significant association of HLA-DR4 or GM could be detected in any of the three categories. When the information of the follow-up investigation was taken into account, a reappraisal of the classification resulted in 53 cases with RA, 18 with Rad only, and 35 with RF only. The frequencies of HLA-DR4 and GM in the three categories were also about the same as those in normal controls. However, an increase in the frequency of HLA-DR4 was observed in cases of RA positive for Rad, RF, or both. We found no evidence for an interaction between HLA-DR4 and GM. Our results suggest that rheumatoid arthritis is a heterogeneous disorder, only a fraction of which is associated with HLA-DR4. At present no single determinant of RA such as Rad or RF can characterise the HLA-DR4-associated, and most probably more severe, type of RA.

It is now well established that rheumatoid arthritis (RA) is associated with an increase in frequency of the antigen HLA-DR4 of the human major histocompatibility complex (MHC). ${ }^{1-3}$ However, the mechanism of that association is not known. One of the problems in studying the genetic factors in RA is that RA represents a clinical diagnosis of a disorder with different environmental and genetic components. ${ }^{4}$ To determine the relationship of HLA-DR4 and disease susceptibility for RA several investigators have tried to restrict their studies to subgroups of RA. The frequency of HLA-DR4 is higher among severe and progressive RA than

Accepted for publication 20 February 1984.

Correspondence to B. M. de Jongh, Department of Immunohaematology and Blood Bank, University Hospital Leiden, Rijnsburgerweg 10, 2333 AA Leiden, The Netherlands. among the milder and stable forms of RA. ${ }^{5}$ However, two studies could not detect an association between HLA-DR4 and severity of RA. ${ }^{36}$ The presence of rheumatoid factor ( $R F$ ) in RA ('seropositive RA') is associated with an increase of HLA-DR4 in some studies ${ }^{17}$ but not in others. ${ }^{89}$ The observation of an association between HLADR4 and RF in RA has been the basis for the hypothesis that HLA-DR4 is primarily associated with the synthesis of anti-immunoglobulin antibodies. ${ }^{10}$ However, HLA-DR4 was not associated with the presence of rheumatoid factor in healthy women. ${ }^{11}$ Other genetic factors have been studied in RA, and one study reported an association between RF in RA and GM, a set of allotypic markers of the immunoglobulin $G$ heavy chains. ${ }^{12}$

The difficulty of relating HLA-DR4 to subgroups 
of RA in relation to the clinical course of the disease or the presence of RF, is that seropositive $R A$ is in itself associated with the more severe form of the disease. ${ }^{13}{ }^{14}$ In addition, most of these studies refer to cases of RA collected from hospital records. This introduces a selection of cases with RA, since many of the less severe cases will not come to the attention of the hospital physician. The result of this selection is difficult to estimate, because little is known about the immunogenetic factors involved in RA and its determinants in the population. We therefore, investigated the frequency of HLA-DR4 and GM allotypes of immunoglobulins in relation to RA as detected in an open Dutch population of 6584 individuals. In addition we typed non-RA cases that were either positive for rheumatoid factor or had erosive abnormalities on radiological examination.

\section{Materials and methods}

Population. The present study was part of the EPOZ study of the Department of Epidemtology of the Erasmus University in Rotterdam. EPOZ is a Dutch acronym for Epidemiological Preventive Investigation (Onderzoek) Zoetermeer, a prevalence survey of chronic diseases and their determinants in 10532 inhabitants of a single community in the western part of The Netherlands. From April 1975 to May 1978 a survey was performed of different rheumatic diseases and their determinants. Of 8700 persons older than 19 years $76 \%$ responded to this survey. As a result 6584 persons (3493 females and 3091 males) were investigated.

Clinical investigation. Each respondent, to whom a questionnaire was sent, was seen by one of four medical doctors trained in rheumatology. The medical history was followed by a systematic examination of the joints, and the results were coded on standard mark sensing sheets, suitable for computer analysis. The clinical diagnoses were coded at the end of the examination without prior knowledge of the results of the radiological and serological investigations.

Radiological investigation. Radiological examination of the hands, feet, and cervical spine was performed on every person. Radiographs were taken of pelvis, lumbar spine, and knees of persons older than 44 years. The radiographs were graded for erosive abnormalities independently by two observers according to the Atlas of Standard Radiographs. ${ }^{15}$ In the event of discrepant readings a final decision was reached in a combined session.

Rheumatoid factor. Serum was investigated for IgM rheumatoid factor by a human erythrocyte agglutination test ${ }^{16}$ and a latex fixation test. ${ }^{17}$ The result was expressed as the number of double titration tubes with a positive reaction.

Data processing and classification. The data were converted in a system file to a form suitable for analysis with the programs of the Statistical Package for the Social Studies (SPSS). ${ }^{18}$ The disease classification was standardised according to the American Rheumatism Association (ARA) criteria, ${ }^{19}$ New York Criteria (NY), ${ }^{20}$ and Manchester Grading (MG). ${ }^{21}$

Follow-up investigation. In 1981 a follow-up investigation was performed, the main follow-up interval averaging five years. Three categories of cases were reinvestigated: (1) cases with clinical RA; (2) non-RA cases with only erosive abnormalities on radiological examination; (3) non-RA cases, positive only for RF.

The criteria for assignment to the three categories were as follows. Category 1: Clinical RA-ARA score of 3 or more (probable and definite, including classical RA), or New York score of 3 or 4 , or Manchester grading of 2 or more. Category 2: Cases with only erosive abnormalities in 2 or more joints on radiological examination; maximal ARA score of 2 of which one criterion was pain. Category 3: cases with positive rheumatoid factor in a human erythrocyte agglutination test and/or latex-fixation test and maximal ARA score of 2 , of which one criterion was pain.

The clinical and laboratory investigations were the same as in 1975-8, with the addition of typing for HLA and GM. The two doctors performing the clinical investigations also participated in the first investigation. In both studies the clinical classification was primarily according to the Manchester grading, without knowledge of the status of the respondent and without information on serology and radiology.

$H L A$ and $G M$ typing. HLA-A, $-\mathrm{B},-\mathrm{C}$ antigens were determined with the standard microcytoxicity assay $^{22}$ on lymphocytes isolated from heparinised blood samples. Typing for HLA-DR antigens was done with the two-colour fluorescence test ${ }^{23}$ using a set of 120 allo-antisera. The $\mathrm{Gm}$ allotypic markers of immunoglobulin heavy chains were determined with the haemagglutination-inhibition assay. ${ }^{24}$

Statistical analysis. The statistical analysis of the HLA and GM antigen frequencies in the cases and controls was performed by the method of Woolf as modified by Haldane. ${ }^{25} \mathrm{~A}$ randomly selected panel of 201 and 792 normal Dutch Caucasians was used as a control for the HLA and GM antigens respectively. ${ }^{26}$ HLA data of hospital patients with classical or definite RA in The Netherlands were derived from two published studies. One study consisted of 82 Dutch Caucasian patients investi- 
gated in Amsterdam ${ }^{27}$ and a second study consisted of 68 patients studied in Nijmegen. ${ }^{28}$ The HLA typings of these two Dutch laboratories and the Leiden Laboratories are checked in regular tissue typing quality control studies for Eurotransplant and show a high degree of concordance.

\section{Results}

In the initial population survey 6584 persons $(3493$ females and 3091 males) older than 19 years were investigated for rheumatic diseases and their determinants. Cases were assigned to one of three categories. Category one consisted of 124 cases (33 males and 91 females) with clinical rheumatoid arthritis as defined by the various sets of criteria. Category two consisted of 44 cases (18 males and 26 females) with only erosive abnormalities on radiological examination (Rad). Category three consisted of 83 cases ( 41 males and 42 females) positive only for RF.

The individuals belonging to the three categories (in total 251 cases) were invited to participate in a follow-up investigation in 1981. The mean follow-up interval was five years. Eventually 200 cases were available, and in 161 cases a clinical investigation and HLA and GM typing could be performed. The details of the 90 cases lost in the follow-up investigation are shown in Table 1. Mortality - as expected in this age category - was somewhat higher for men than for women, but no significant differences occurred among the three categories. Slightly more women with RA moved out of the area, mostly into homes for the aged. In all categories approximately $80 \%$ were available for the five-year follow-up investigation, and of that group between 71 and $88 \%$ responded to the second study. No evidence was found for a selective loss of severe cases of RA in category one.

The final 161 cases consisted of 83 cases ( 20 males and 63 females) with clinical RA, 30 cases (12 males and 18 females) with only erosive abnormalities, and 48 cases ( 24 males and 24 females) with only RF. Of 83 cases with RA (category one), $19(23 \%)$ were classified as having definite RA and $64(77 \%)$ as having probable RA; 17 cases $(21 \%)$ of RA had erosive arthritis and 23 cases $(28 \%)$ of RA were seropositive. The female to male ratio was $3: 1$ among the cases with RA. Although the median age for men was slightly lower than for females with RA, no significant differences in median age were observed for sex in the three categories. A report of the detailed diagnostic findings in the population survey and the follow-up investigation is in preparation.

The first analysis was done with classification of the cases based on the findings of the initial population survey in 1975-8. The frequency of HLA-DR4 in the three categories was not significantly different from that in normal controls. Only a slight increase in frequency of HLA-DR4 was observed in the cases with clinical RA (HLA-DR4 total $28 \%$; male $30 \%$, female $27 \%$ ). This result was in contrast with a significant increase in frequency of HLA-DR4 as reported in cases of RA in two clinical studies in The Netherlands. The relative risk of HLA-DR4 and RA in the population was significantly different from that in the hospital series of Nijmegen and Amsterdam; $\chi^{2}(1 \mathrm{DF})$ was 10.9 and 9.6 respectively (for method of calculation see Svejgaard et al. ${ }^{25}$ ), whereas no significant differences were observed in the HLA-DR frequencies of the normal control populations used in the respective studies. In the cases positive only for RF we observed a slight decrease in frequency of HLADR4 as compared with the normal controls (HLADR4 total $19 \%$; male $30 \%$, female $8 \%$ ). The difference in the frequency of HLA-DR4 between male and females was not significant $\left(\chi^{2}=2 \cdot 99\right)$. No other HLA-A, $-\mathrm{B},-\mathrm{C}$ and $-\mathrm{DR}$ antigens were significantly different in frequency as compared with normal controls. In the cases with only erosive abnormalities on radiological examination a slight increase in frequency of HLA-DR4 was observed (HLA-DR4 total 33\%; male 33\%, female 33\%).

The frequencies of GM were analysed per allotype, and no significant differences in frequencies in the three categories were observed as compared with normal controls, and no significant differences in the two sexes were detected (data not shown). GM results for the hospital cases of RA were not available for comparison.

In summary, the results of the first analysis showed that HLA-DR4 was not or was only weakly associated with RA as detected in an open Dutch population. This result was in marked contrast with the association of HLA-DR4 and RA as observed in two Dutch hospital series. In addition the GM phenotype frequencies among the three categories did not differ significantly from those in normal controls.

Since the definition of RA is based on criteria that fluctuate considerably in time, we restricted the second analysis to those cases that were positive for the criteria of one of the three categories in both the initial population survey and in the follow-up investigation. After the follow-up study was concluded a reappraisal of the material was performed by one of us (H.A.V.). The reappraisal was necessary because some patients had changed their clinical status in the five years. In addition a considerable number of cases selected on the basis 


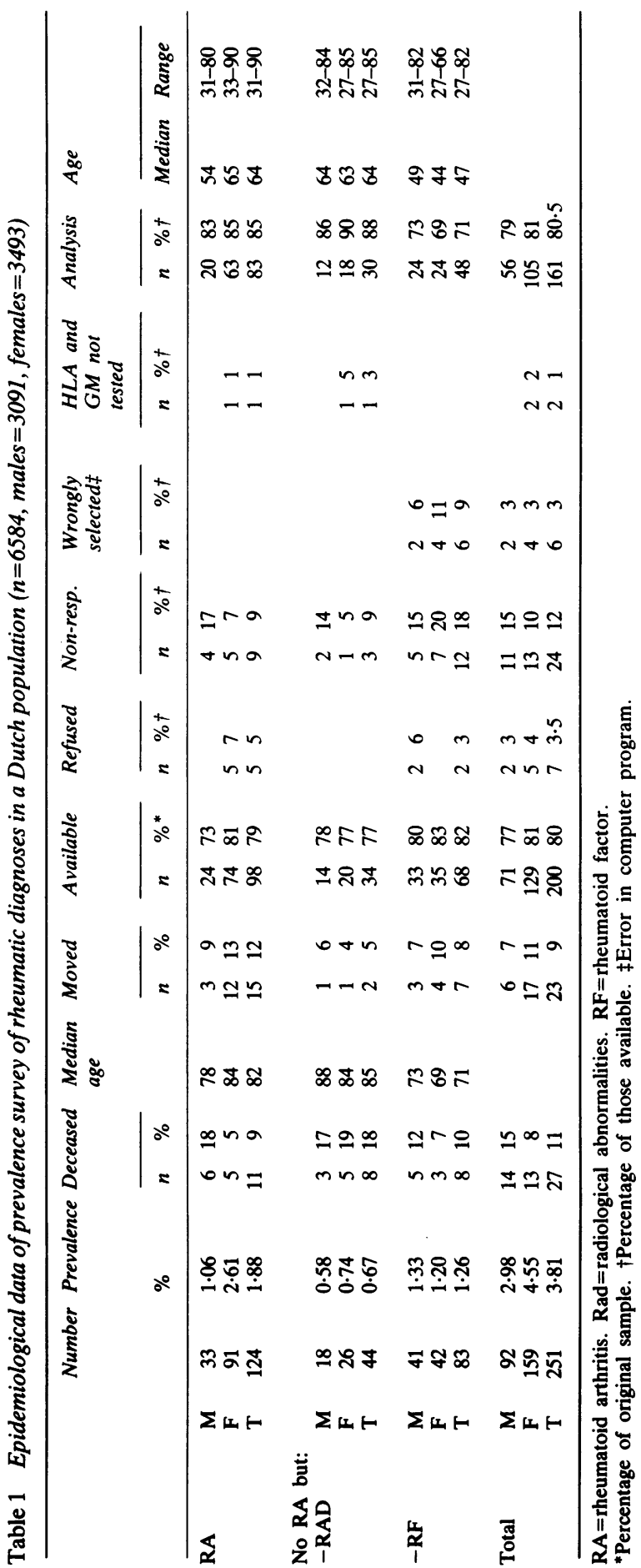


of the New York criteria 1 and 2 turned out to have localised or generalised osteoarthrosis and not RA. As a result, 53 cases were classified in category 1 with RA. Category 2 consisted of 18 cases with only erosive abnormalities on radiological examination, and category 3 consisted of 35 cases positive only for RF. Table 2 shows the frequency of HLA-DR4 in the various categories. An increase in the frequency of HLA-DR4 was observed among the cases with RA, and especially in those that were positive for $\mathrm{Rad}$, RF or both. However, those increases were not significant, given the low number of cases in each category. In the non-RA cases positive only for Rad or RF no significant differences in the frequency of HLA-DR4 were observed as compared with normal controls. Of the 53 cases with clinical RA 17 were HLA-DR4 positive. To determine whether or not HLA-DR4 was a marker for a subgroup of RA we compared the 17 HLA-DR4 positive cases in category 1 with the 36 HLA-DR4 negative cases in the same category. According to the ARA criteria 41 versus $50 \%$ had probable RA, 35 versus $40 \%$ definite, and 24 versus $8 \%$ had classical RA. These differences were not significantly different. The same was true for differences in other parameters such as age, sex, frequency of Rad and RF, mean RA score, and Manchester grading, as will be described in detail in another paper (in preparation).

Table 3 presents the data of four GM phenotypes among the cases in the various categories after reappraisal of the diagnosis. In addition we divided the cases with RA in those that were HLA-DR4 positive and that were HLA-DR4 negative. No significant differences in the frequencies of the separate allotypes were observed among the various categories, nor were the differences significant when GM and HLA-DR4 were considered jointly.

\section{Discussion}

Although it is well established that rheumatoid arthritis is associated with an increase in frequency of HLA-DR4, the mechanism of this association is unknown. Various investigators have tried to subdivide groups of RA that would in particular be associated with HLA-DR4. The results of these subdivisions, however, have been conflicting. Common to most of these studies is that cases of RA were ascertained from hospital records. The hospital cases of RA are likely to be a selection of the more severe cases of RA that occur in the population, and little is known of the relation of RA and its determinants in the population with genetic markers

Table 2 Frequency of HLA-DR4 among categories of rheumatic diagnoses

\begin{tabular}{|c|c|c|c|c|c|c|c|}
\hline & \multicolumn{3}{|c|}{ Cases } & \multicolumn{3}{|c|}{ Controls } & \multirow[t]{2}{*}{$p$} \\
\hline & $n$ & DR4 pos & $\%$ & $n$ & DR4 pos & $\%$ & \\
\hline $\begin{array}{l}\text { RA total } \\
\text { RA + Rad and/or RF } \\
\text { Rad but no RA } \\
\text { RF but no RA }\end{array}$ & $\begin{array}{l}53 \\
16 \\
18 \\
35\end{array}$ & $\begin{array}{r}17 \\
6 \\
5 \\
6\end{array}$ & $\left.\begin{array}{l}32 \\
38 \\
28 \\
17\end{array}\right\}$ & 201 & 49 & $24 \cdot 4$ & $\begin{array}{l}\text { NS } \\
\text { NS } \\
\text { NS } \\
\text { NS }\end{array}$ \\
\hline $\begin{array}{l}\text { Ref. } 28 \text { clinical RA } \\
\text { Ref. } 27 \text { clinical RA }\end{array}$ & $\begin{array}{l}66 \\
82\end{array}$ & $\begin{array}{l}36 \\
57\end{array}$ & $\begin{array}{l}55 \\
69\end{array}$ & $\begin{array}{r}277 \\
84\end{array}$ & $\begin{array}{l}54 \\
26\end{array}$ & $\begin{array}{l}19 \cdot 6 \\
31\end{array}$ & $\begin{array}{l}0.01 \\
0.001\end{array}$ \\
\hline
\end{tabular}

$\mathbf{R A}=$ rheumatoid arthritis. $\mathrm{Rad}=$ radiological abnormalities. $\mathrm{RF}=$ rheumatoid factor. $\mathrm{NS}=$ not significant.

Table 3 Comparison of GM phenotypes in categories of rheumatic diagnoses and normal controls

\begin{tabular}{|c|c|c|c|c|c|c|c|c|c|}
\hline & \multicolumn{3}{|c|}{$f b$} & \multicolumn{2}{|l|}{$f n b$} & \multicolumn{2}{|l|}{$z a g$} & \multicolumn{2}{|c|}{$z a x g$} \\
\hline & $n$ & pos & $\%$ & pos & $\%$ & pos & $\%$ & pos & $\%$ \\
\hline Normal controls & 792 & 161 & $20 \cdot 3$ & 542 & $68 \cdot 4$ & 228 & $28 \cdot 8$ & 151 & $19 \cdot 1$ \\
\hline RA total & 53 & 7 & 13 & 38 & 72 & 19 & 36 & 9 & 17 \\
\hline $\mathbf{R A}+\mathbf{R a d}$ and/or RF & 16 & 2 & 13 & 13 & 81 & 6 & 38 & 3 & 19 \\
\hline RA, DR4 pos. & 17 & 1 & 6 & 13 & 76 & 5 & 29 & 2 & 12 \\
\hline Rad. but no RA & 18 & 4 & 22 & 11 & 61 & 5 & 28 & 3 & 17 \\
\hline RF, but no RA & 35 & 8 & 23 & 24 & 69 & 4 & 11 & 8 & 23 \\
\hline
\end{tabular}

$\mathbf{R A}=$ rheumatoid arthritis. Rad=radiological abnormalities. $\mathbf{R F}=$ rheumatoid factor. 
such as HLA-DR and GM. The present study investigated these genetic markers in cases positive for RA or its determinants as detected in a prevalence survey of 6584 Dutch persons older than 19 years and living in a single community in the western part of The Netherlands.

The results of this investigation revealed that no significant association could be detected between HLA-DR4 or GM and cases of RA in the population nor with cases positive only for Rad or RF. What are the possible explanations for the discrepancy in the association of HLA-DR4 in the hospital versus the population cases of RA? In the interpretation of these results we will consider the following aspects.

First, 41 cases of RA were not available for the follow-up study and could not be typed for the genetic markers. If these 41 cases were not a random sample of the original 124 cases of RA, then a selection bias could have been introduced in the final sample available for analysis. However, no evidence was found for a selective loss of cases with RA as related to age, sex, or disease. Secondly, the ethnic background of the community could have been different from the areas where the clinical cases of RA had been described. However, the Dutch population is fairly homogeneous in this respect, and within the Netherlands the various studied normal control groups are homogeneous with respect to their HLA antigen frequencies. In addition a group of 50 normal controls from the EPOZ community studied for HLA confirmed this observation (data not shown). Thirdly, the age and sex composition of the cases with RA might be a confounding factor, but our results are in agreement with those of other epidemiological investigations in Caucasian patients with RA. ${ }^{21}$

Finally, an important consideration relates to the criteria for RA as used in the disease classification. At present the diagnosis of RA is based on the accepted standard criteria that are known as the ARA, New York, and Manchester criteria. ${ }^{19-21}$ Common to these criteria is a probability factor, illustrated by categories as 'possible', 'probable', and 'definite'. Shifts of cases among these categories is a well-known observation among rheumatologists. The probability factor a fortiori applies to epidemiological studies, as illustrated by the Sudbury study, ${ }^{29}$ because cases with a lower probability for RA are also detected.

In the present study we were able to carry out a five-year follow-up. After reappraisal of the total material we observed no significant association between HLA-DR4 and RA in 53 cases twice classified as having RA. A subdivision of these cases in those that were twice positive for Rad, RF, or both revealed that the frequency of HLA-DR4 was increased, though not significantly. A reverse approach compared HLA-DR4 positive with HLADR4 negative cases of RA. But the preliminary results of this analysis, which will be further reported, revealed no statistically significant differences according to various disease parameters.

A different aspect that we studied was the investigation of cases in the population positive only for determinants of RA, such as the presence of RF or erosive abnormalities on radiological examination. In particular we considered presence of RF in otherwise normal individuals, since it was suggested that HLA-DR4 was associated particularly with RA cases positive for RF. However, we found no increase in frequency of HLA-DR4 in normal cases with IgM RF. Thus we were able to confirm in a larger series the observation of Engleman et al. ${ }^{11}$

Finally, the analysis of the GM phenotypes in the various categories revealed no significant associations nor provided evidence for an interaction or association between HLA-DR4 and GM.

Our overall results suggest the following conclusions. The absence of a significance association between HLA-DR4 and RA detected in the population, as compared with the association observed in clinical cases, suggests that RA is a heterogeneous disease. Moreover, the presence of Rad or RF as determinants for RA is not in itself responsible for the association with HLA-DR4. Consequently other and as yet unknown disease factors may be implicated in RA as seen in hospitals. In this regard the presence of HLA-DR4 might be associated with a disease-modifying factor rather than with a disease-susceptibility factor. In agreement with this hypothesis is a recent observation that HLA-DR4 was associated with a more severe disease in seronegative RA, which in itself was not associated with HLA-DR4 (30). Finally our results suggest that future studies of RA will benefit from an approach that compares, in detail, the HLA-DR4 positive with HLA-DR4 negative cases of RA for various clinical and laboratory parameters. In this regard the severe form of RA, known as the Felty's syndrome, with a strong association of HLA-DR4 ${ }^{27}$ might provide a lead to the pathogenetic process involved in HLA-DR4-positive RA.

We thank Mrs $\mathrm{C}$. Valkenburg and $\mathrm{H}$. de Bruin for their expert help in organisation of the follow-up investigation, Ir. G. M. Th. Schreuder and co-workers for performing the HLA typings, Dr F. Klein for the rheumatoid factor determination, $\operatorname{Dr} R$. R. P. de Vries, and Professor A. Cats for their valuable suggestions.

This work was supported in part by the Dutch Foundation for Medical Research (FUNGO), which is subsidised by the Dutch Organisation for the Advancement of Pure Research (ZWO), the J 
A Cohen Institute for Radiopathology and Radiation Protection (IRS), and the Prevention Fund.

\section{References}

1 Stastny P. Mixed lymphocyte culture typing cells from patients with rheumatoid arthritis. Tissue Antigens 1974; 4: 571-9.

2 Thomsen M, Morling N, Snorrason E, Svejgaard A, Sorensen S F. HLA-Dw4 and rheumatoid arthritis. Tissue Antigens 1979; 13: $56-60$

3 Stastny P. Rheumatoid arthritis. Joint report. In: Terasaki P I, ed. Histocompatibility testing 1980. Los Angeles: UCLA Tissue Typing Laboratory, 1980: 681-6.

4 Bennett J C. The etiology of rheumatoid arthritis. In: Kelly W N, Harris E D, Ruddy S, Sledge C B, eds. Textbook of rheumatology. Philadelphia: Saunders, 1981; i: 887-95.

5 Roitt I, Festenstein H, Jaraquemada Papasteriadis C, Hay F C, Nineham L J. HLA-DRw4 and prognosis in rheumatoid arthritis. Lancet, 1978; i: 990.

6 Husby G, Gran J T, Østensen M, Johannessen A, Thorsby E. HLA-DRw4 and rheumatoid arthritis. Lancet, 1979; i: 549.

7 Dobloug J H, Forre O, Kass E, Thorsby E. HLA antigens and rheumatoid arthritis: association between HLA DRw4 possitivity and IgM rheumatoid factor production. Arthritis Rheum 1980; 23: 309-13.

8 Panayi G S, Wooley P H, Batchelor J R. HLA-DRw4 and rheumatoid arthritis. Lancet, 1979; i: 730.

9 Scherak O, Smolen J S, Mayr W R. Rheumatoid arthritis and lymphocyte-B alloantigen HLA-DRw4. J Rheumatol 1980; 7: 9-12.

10 Stastny P. Immunogenetic factors in rheumatoid arthritis. Clin Rheum Dis 1977; 3: 315-32.

11 Engleman E, Sponzilli E E, Batey M E, Ramchran S, McDevitt $H$ O. Mixed lymphocyte reaction in healthy women with rheumatoid factor: lack of association with HLA-Dw4. Arthritis Rheum 1978; 21: 690-3.

12 Propert D N, Kay P, McKluskey J, Zilko P J, Mathews J. Immunoglobulin allotypes in rheumatoid arthritis. In: Dawkins R L, Christiansen F T, Zilko P J, eds. Immunogenetics of rheumatoid arthritis. Amsterdam: Excerpta Medica, 1982: after 3:13.

13 Roitt I M, Hay F C, Nineham L J, Male D K. Rheumatoid arthritis In: Lachman P J, Peters D K, eds. Clinical aspects of immunology. Oxford: Blackwell, 1982; 2: 1161-95.

14 Cats A, Hazevoet H M. Significance of positive tests for rheumatoid factor in the prognosis of rheumatoid arthritis. Ann Rheum Dis 1970; 29: 254-60.
15 Atlas of standard radiographs of arthritis. In: Kellgren $\mathbf{J} \mathbf{H}$, Jeffrey M R, Ball J, eds. The epidemiology of chronic rheumatism. Oxford: Blackwell, 1963: 2.

16 Valkenburg H A. The human erythrocyte agglutination test. In: Kellgren J H, Jeffrey M R, Ball J eds. The epidemiology of chronic rheumatism. Oxford: Blackwell, 1963; i: 330.

17 Valkenburg H A. The latex fixation test. In: Kellgren J H, Jeffrey M R, Ball J, eds. The epidemiology of chronic rheumatism. Oxford: Blackwell, 1963; i: 337.

18 SPSS. In: Nie N H, Hull H, Jenkins J G, Steinbrenner K, Bent D H, eds. SPSS: statistical package for the social sciences. 2nd ed. New York: McGraw-Hill, 1975.

19 Ropes M W, Bennet G A, Cobb S, Jacox R, Jessar R. 1958 Revision of diagnostic criteria for rheumatoid arthritis. Bull Rheum Dis 1958; 9: 175-6.

20 Bennett P H, Burch T A. New York symposium on population studies in the rheumatic diseases: new diagnostic criteria. Bull Rheum Dis 1967; 17: 453-8.

21 Kellgren J H, Lawrence J S. Rheumatoid arthritis in a population sample. Ann Rheum Dis 1956; 15: 1-11.

22 Van Rood J J. Microlymphocytotoxicity method. In: John G Ray ed. NIAID Manual of tissue typing techniques. Maryland, USA: NIH 80-545, 1979: 104-05.

23 Van Leeuwen A, Van Rood J J. Description of the B cell method. In: Terasaki P I ed. Histocompatibility testing 1980. Los Angeles: UCLA Tissue Typing Laboratory 1980: 278-79.

24 Van Loghem E. Genetic studies on human immunoglobulins In: Weir D M, ed. Handbook of experimental immunology. 3rd ed. Oxford: Blackwell, 1978; I (Immunochemistry): 11·1-11·16.

25 Svejgaard A, Jersild C, Staub Nielsen L, Bodmer W F. HLA antigens and disease; statistical and genetical considerations. Tissue Antigens 1974; 4: 95-105.

26 Fraser G R, Volkers W S, Bernini L F, Van Loghem E, Meera Khan P, Nijenhuis L E. Gene frequencies in a Dutch population. Hum Hered 1974; 24: 435-48.

27 Dinant H J, Hissink Muller W, Van den Berg-Loonen E M, Nijenhuis L E, Engelfriet C P. HLA-DRw4 in Felty's syndrome. Arthritis Rheum 1980; 23: 1336.

28 Speerstra F, Reekers P, Van De Putte L B A, Vandenbroucke $J$ P, Rasker J J. HLA-DR antigens and proteinuria induced by aurothioglucose and D-penicillamine in patients with rheumatoid arthritis. J Rheumatol 1983; 10: 948-54.

29 O'Sullivan J B, Cathcart E S. Prevalence of rheumatoid arthritis: Follow up evaluation of the effect of criteria on rates in Sudbury, Massachusetts. Ann Intern Med 1972; 76: 573-7.

30 Alarcon G S, Koopman W J, Acton R T, Barger B O. Seronegative rheumatoid arthritis. A distinct immunogenetic disease? Arthritis Rheum 1982; 25: 502-7. 